\title{
Zirconium Zr 89 Nanocolloidal Albumin
}

National Cancer Institute

\section{Source}

National Cancer Institute. Zirconium Zr 89 Nanocolloidal Albumin. NCI Thesaurus. Code C131498.

A radioimmunoconjug ate composed of a nanoformulation of colloidal human serum albumin (HSA) labeled with zirconium Zr 89 (Zr89), via the bifunctional chelate pisothiocyanatobenzyldesferrioxamine $B$, with potential imaging activity using positron emission tomography (PET). Upon administration, the zirconium Zr 89 nanocolloidal albumin travels through the bloodstream and into the lymphatic system. Using PET, the draining pattern of the lymph nodes can be visualized and lymph node status, including that of the sentinel lymph node (SLN), can be detected and analyzed. Compared to some other formulations, the smaller particle size of nanocolloidal albumin allows for enhanced imaging of the lymphatic system. 